\title{
Transdifferentiation of human MNNG/HOS osteosarcoma cells into vascular endothelial cells in vitro and in vivo
}

\author{
XINWEN WANG ${ }^{1}$, WEIFENG XU ${ }^{1,2}$, SHENGLIN WANG $^{1}$, FEQIANG YU $^{1}$, JINYI FENG $^{3}$, \\ XINWU WANG ${ }^{4}$, LURONG ZHANG ${ }^{1,2,5}$ and JIANHUA LIN ${ }^{1}$
}

\begin{abstract}
${ }^{1}$ The First Affiliated Hospital of Fujian Medical University; ${ }^{2}$ Fujian Key Laboratory of Individualized Active Immunotherapy, Fuzhou, Fujian 350005; ${ }^{3}$ Department of Orthopaedics, The First Affiliated Hospital of Xiamen University, Xiamen, Fujian 361003; ${ }^{4}$ Department of Orthopaedics, The First Hospital of Putian City, Putian, Fujian 351100, P.R. China;

${ }^{5}$ Department of Radiation Oncology, University of Florida, Gainesville, FL 32610, USA
\end{abstract}

Received March 30, 2017; Accepted August 25, 2017

DOI: 10.3892/or.2017.6005

\begin{abstract}
The transdifferentiation of cancer cells into other types of cells in several types of tissues or organs has been studied. However, whether human osteosarcoma MNNG/HOS cells can transdifferentiate into other types of cells has seldom been reported. Meanwhile, the mechanism of tumor angiogenesis is still disputed, and whether MNNG/HOS cells participate in angiogenesis in osteosarcoma remains unknown. In the present study, the investigation was divided into two parts: in vitro and in vivo. In vitro, we cultivated MNNG/HOS cells under hypoxic conditions for 4 days and found that they typically showed a characteristic 'flagstone' appearance as cultured vascular endothelial cells (VECs). MNNG/HOS cells that were cultivated on Matrigel under hypoxic conditions gradually formed tubular-like structures. Furthermore, when cultured under hypoxic conditions for 4 days, MNNG/HOS cells also transcribed and expressed several molecular markers of VECs (CD31, CD34 and vWF). In vivo, MNNG/HOS cells $\left(1 \times 10^{6}\right.$ cells) were cultivated under hypoxic conditions and subcutaneously injected into nude mice; the mice were sacrificed 49 days after inoculation. Immunohistochemical staining with anti-human CD31 antibody showed evidence of tumor angiogenesis in human osteosarcoma MNNG/HOS cells. The results demonstrated that MNNG/HOS cells can transdifferentiate into vascular endothelial cell-like cells in vitro and in vivo.
\end{abstract}

\section{Introduction}

Osteosarcoma is the most common type of solid bone cancer, mainly arising in children and young adults (1). The majority

Correspondence to: Professor Jianhua Lin, Department of Orthopedics, The First Affiliated Hospital of Fujian Medical University, 20 Chazhong Road, Fuzhou, Fujian 350005, P.R. China E-mail: jhlin_fyyy@126.com

Key words: osteosarcoma cells, transdifferentiation, hypoxia, vascular endothelial cells of osteosarcomas develop in the long bones, most commonly in the distal femur and proximal tibia (2). It is highly malignant and invasive, with a metastatic rate of $20 \%$ (3). Other common targets are the lung and other bones in addition to long bones $(4,5)$. Numerous studies have reported that angiogenesis is closely related to the development, invasion and metastasis of osteosarcoma (6). Therefore, actively exploring the mechanism of angiogenesis in osteosarcoma may help to enhance understanding of the mechanisms of osteosarcoma occurrence and progression and potentially aid in developing a new strategy for anti-angiogenesis clinical tumor treatment.

Tumor angiogenesis involves degradation of the extracellular matrix, endothelial cell (EC) migration, proliferation, elongation and tube formation to form new vessels (7). Pre-existing ECs, bone marrow-derived endothelial progenitor cells (EPCs) and mesenchymal stem cells have traditionally been regarded as the sources of ECs in tumor angiogenesis $(8,9)$. However, recent studies suggest that tumor cells may differentiate into ECs in myeloma, lymphoma, chronic myeloid leukemia (CML), glioblastoma, breast cancer and neuroblastoma (10-15). However, whether the mechanism of tumor angiogenesis in osteosarcoma is also different from regular tumor vascular formation, remains an unanswered question. The aim of the present study was to determine whether human osteosarcoma MNNG/HOS cells can also transdifferentiate into ECs and acquire endothelial markers in vitro, and whether they participate in tumor vascularization in vivo.

For this purpose, the research is divided into two parts: in vitro and in vivo. In vitro, we cultured MNNG/HOS cells on Matrigel under hypoxic conditions, and then investigated changes in the morphology and molecular phenotype of the cells. In vivo, human osteosarcoma cells $\left(1 \times 10^{6}\right.$ cells $)$ that were cultured under conditions of hypoxia were subcutaneously injected into nude mice, and then the mice were sacrificed 49 days after inoculation. Tumor angiogenesis in human MNNG/HOS osteosarcoma cells xenografted in nude mice was confirmed using immunohistochemical staining with anti-human CD31 antibody. The results demonstrated that human MNNG/HOS osteosarcoma cells can transdifferentiate into vascular endothelial cell-like cells in vitro and in vivo. 


\section{Materials and methods}

Cell lines and culture. The human osteosarcoma cell line MNNG/HOS was obtained from the Cell Bank of the Chinese Academy of Sciences (Shanghai, China). The cells were cultured in essential medium MEM $+10 \%$ fetal bovine serum (FBS) (both from HyClone, Logan, UT, USA) + $100 \mathrm{U} / \mathrm{ml}$ penicillin/streptomycin in vitro.

Induced transdifferentiation of human osteosarcoma cells. The MNNG/HOS cells were divided into 4 groups: control, normoxia, hypoxia and preconditioning group. MNNG/HOS cells in the control and preconditioning group were cultured in essential medium. MNNG/HOS cells in the normoxia and hypoxia group were cultured in endothelial differentiation medium consisting of MEM/F-12 containing 10\% FBS (HyClone), 1\% N2 supplement (Gibco, Carlsbad, CA, USA), human vascular endothelial growth factor $(20 \mathrm{ng} / \mathrm{ml})$, human recombinant epidermal growth factor $(20 \mathrm{ng} / \mathrm{ml})$ (both from Invitrogen, Carlsbad, CA, USA), basic fibroblast growth factor (10 ng/ml) and heparin (both from Gibco) in vitro. MNNG/HOS cells in the control and normoxia group were cultivated in an incubator at $37^{\circ} \mathrm{C}$ under conditions of normoxia $\left(5 \% \mathrm{CO}_{2}, 95 \%\right.$ air). MNNG/HOS cells in the hypoxia and preconditioning group were cultivated in an incubator at $37^{\circ} \mathrm{C}$ under conditions of hypoxia $\left(1 \% \mathrm{O}_{2}, 5 \% \mathrm{CO}_{2}, 94 \%\right.$ nitrogen). Four days later, their appearance was observed.

Three-dimensional culture. Matrigel (BD Biosciences, Franklin Lakes, NJ, USA) was dissolved at $4^{\circ} \mathrm{C}$ overnight. Ninety-six well plates were prepared at $4^{\circ} \mathrm{C}$ for $60 \mathrm{~min}$. Matrigel (30 $\mu \mathrm{l})$ was poured onto the 96 -well plates, and then the plates were placed in an incubator at $37^{\circ} \mathrm{C}$ with $5 \% \mathrm{CO}_{2}$ for $30 \mathrm{~min}$. After allowing $30 \mathrm{~min}$ for Matrigel gel formation, MNNG/HOS cell $\left(1 \times 10^{5}\right.$ cells/200 $\left.\mu \mathrm{l}\right)$ suspensions in the endothelial differentiation medium were added to the Matrigel-coated wells. For the control and normoxia group, the cells were cultivated at $37^{\circ} \mathrm{C}$ with $5 \% \mathrm{CO}_{2}$ and $95 \%$ air. For the hypoxia and preconditioning group the cells were cultivated at $37^{\circ} \mathrm{C}$ with $1 \% \mathrm{O}_{2}, 5 \% \mathrm{CO}_{2}$ and $94 \%$ nitrogen. Formation of tubular-like structures and networks was periodically observed and imaged by inverted phase contrast microscopy.

Isolation of RNA and RT-PCR. MNNG/HOS cells in each group were cultivated in different medium under normoxia or hypoxia condition. Forty-eight hours later, total RNA was extracted from each group using TRIzol reagent and treated with DNase I (both from Invitrogen). First-strand cDNA was synthesized from total RNA (5 $\mu \mathrm{g})$ using a ReverTra Ace kit (Toyobo Co., Ltd., Osaka, Japan) and an oligo(dT) ${ }_{20}$ primer. First-strand cDNA was synthesized from total RNA using a ReverTra Ace kit (Toyobo) and an oligo(dT) ${ }_{20}$ primer. The target cDNA was amplified using a combination of Taq DNA polymerase and the proofreading Pfu DNA polymerase. The primers used were: CD31 forward (5'-ACA TGG CAA CAA GGC TGT GTA-3') and reverse (5'-CCT CAA ACT GGG CAT CAT AAG-3'); CD34 forward (5'-CCA CTC GGT GCG TCT CTC TAG GAG C-3') and reverse (5'-TTG TCT CTG GAG TTG AAA CGT TGG C-3'); vWF forward (5'-CTG AAG AGT CAT CGG GTC AAC TGT-3') and reverse
(5'-AGC ATG AAG TCA TTG GCT CCG TTC T-3'); $\beta$-actin forward (5'-TTC TGT GGC ATC CAC GAA ACT-3') and reverse (5'-GAA GCA TTT GCG GTG GAC GAT-3'). PCR amplification was carried out in a final volume of $25 \mu \mathrm{l}$ of reaction mixture. After amplification, $5 \mu \mathrm{l}$ of the PCR products was electrophoresed through a $1.5 \%$ agarose gel.

In vivo xenograft experiments. Five-to-six week-old male $\mathrm{BALB} / \mathrm{c}$ nude mice were obtained from the Chinese Academy of Medical Sciences. Animals were housed in laminar flow cabinets under specific pathogen-free conditions. For the xenograft experiments, mice were randomized into 4 groups, each with 5 mice. MNNG/HOS cells $\left(1 \times 10^{6}\right.$ cells) in each group were respectively subcutaneously injected into the nude mice. After inoculation, the size of the subcutaneous tumors was closely monitored. The tumor volume (V) was calculated by the formula, $\mathrm{V}=0.5\left(\mathrm{~L} \mathrm{x} \mathrm{W}^{2}\right) \mathrm{mm}^{3}$; the longest axis diameter (L) and the greatest transverse diameter (W) were estimated with external calipers every 7 days. The mice were sacrificed 49 days after inoculation, and the tumors were weighed and harvested for further evaluation by immunohistochemistry. The mice were sacrificed and cared for according to the ethical guidelines of the Animal Experimental Ethics Committee of Fujian Medical University. None of the mice died during the whole experimental process.

Immunofluorescence. MNNG/HOS cells were plated onto glass coverslips in 12-well plates and cultivated according to the different conditions of each group. Four days later, the above-mentioned cells were washed with phosphate-buffered saline (PBS) (pH 7.4) and fixed with $4 \%$ paraformaldehyde for $20 \mathrm{~min}$ at $37^{\circ} \mathrm{C}$ and washed 3 times with PBS. Following permeabilization with $0.3 \%$ Triton $\mathrm{X}-100$ for $5 \mathrm{~min}$ at room temperature, the cells were blocked with $1 \%$ bovine serum albumin (BSA) for $20 \mathrm{~min}$ at room temperature. The washed cells were incubated overnight in the dark at $4^{\circ} \mathrm{C}$ with anti-human CD31 (1:200; rabbit anti-human), anti-human CD34 (1:200; mouse anti-human), and anti-human von Willebrand factor (vWF) (1:200; rabbit anti-human) (all from Abcam, Cambridge, MA, USA) antibodies. The cells were stained with PE-conjugated anti-mouse antibodies or FITC-conjugated anti-rabbit antibodies (1:200; Abcam). Cell nuclei were counterstained with 4,6-diamidino-2-phenylindole (DAPI) (Sigma). All fluorescence images were captured using an Olympus BX51 fluorescence microscope (Olympus BX51; Olympus Corp., Tokyo, Japan).

Immunohistochemistry. For immunohistochemistry, the xenograft of mouse tissues was cut into $4-\mu$ m-thick paraffin sections. The tissue sections were incubated with antibodies against human CD31 (1:50; rabbit anti-human; Abcam). The specimens were then incubated with the secondary antibody, an anti-rabbit peroxidase-labeled polymer (1:50; ZSGB-BIO, Beijing, China). The images were captured with light microscopy (1X71 inverted microscope; Olympus Corp., Tokyo).

Statistical analysis. Statistical calculations were performed using SPSS 20.0 and GraphPad software (GraphPad Software, San Diego, CA, USA). The quantitative assays of the statistical analysis are expressed as the means \pm standard error of the 
A

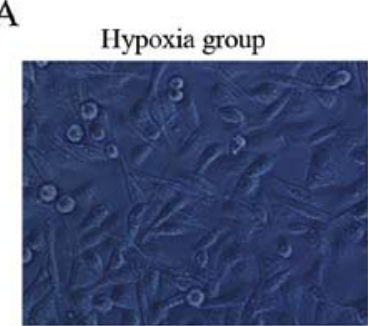

B

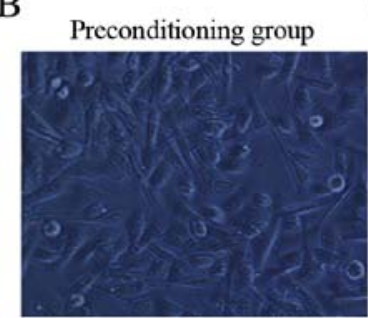

$\mathrm{C}$

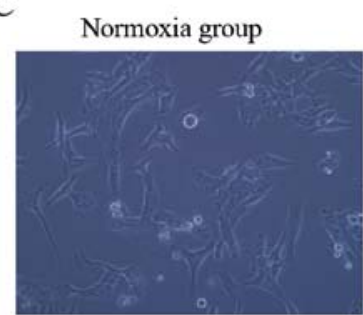

$\mathrm{D}$

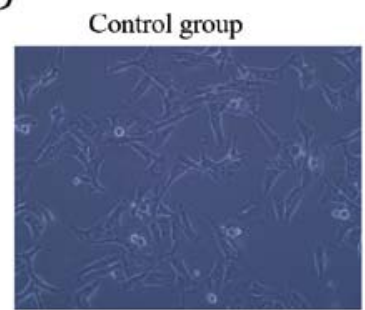

Figure 1. Morphological changes in MNNG/HOS cells. (A) Magnification (x200), the 'flagstone' appearance of MNNG/HOS cells after being cultivated under hypoxia conditions in endothelial differentiation medium for 4 days. (B) Magnification (x200), the 'flagstone' appearance of MNNG/HOS cells after being cultivated under hypoxia conditions in essential medium for 4 days. (C) Magnification (x100), MNNG/HOS cells cultivated under normoxia conditions in endothelial differentiation medium for 4 days. (D) Magnification (x100), MNNG/HOS cells cultivated under normoxia conditions in essential medium for 4 days.

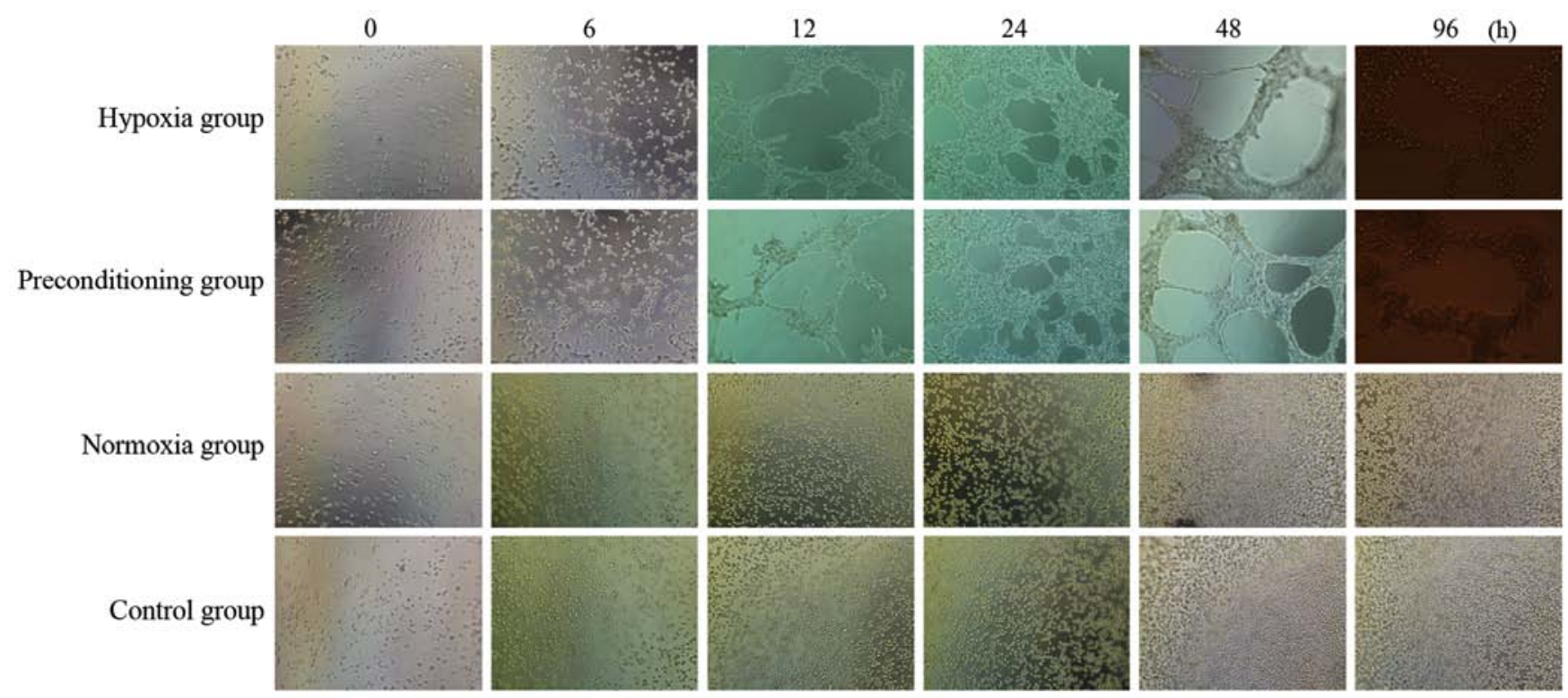

Figure 2. Serial morphological changes in MNNG/HOS cells cultivated on Matrigel for each group. Representative images show the tube network formed 0, $6,12,24,48$ and $96 \mathrm{~h}$ after seeding.

mean. The independent-sample t-tests or one-way analysis of variance were used to determine statistical comparisons. The asterisks in the figures indicate statistically significant differences between the samples $(\mathrm{P}<0.05)$, while 'NS' indicates that there was no statistically significant difference between samples.

\section{Results}

MNNG/HOS cells have morphological features of VECs after transdifferentiation. Four days after MNNG/HOS cells were cultured under hypoxia conditions, the hypoxia and preconditioning group typically developed a characteristic 'flagstone' appearance (Fig. 1A and B). However, for MNNG/HOS cells cultured under normoxia conditions, the control and normoxia group adhered to the culture dish rather than developing a 'flagstone' appearance (Fig. 1C and D). When we cultivated MNNG/HOS cells on Matrigel under hypoxia conditions, the hypoxia and preconditioning group underwent a range of changes: from a single or several cells $(0 \mathrm{~h})$, to intercellular connections or discontinuous net-like connections $(6 \mathrm{~h})$, to continuous net-like connections (12 h),

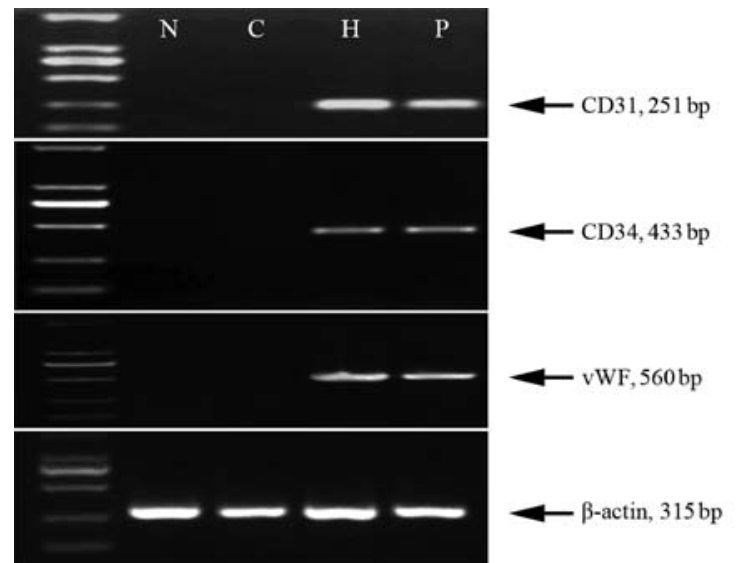

Figure 3. Changes in mRNA of CD31, CD34 and vWF in MNNG/HOS cells after exposure to different culture conditions, as detected by RT-PCR (N, normoxia; $\mathrm{C}$, control; $\mathrm{H}$, hypoxia; and $\mathrm{P}$, preconditioning group).

to the significant increase in the number of tubes $(24 \mathrm{~h})$, to a significant increase in the branch length $(48 \mathrm{~h})$. Vascular mimicry (VM) still existed at $96 \mathrm{~h}$ after seeding. However, 


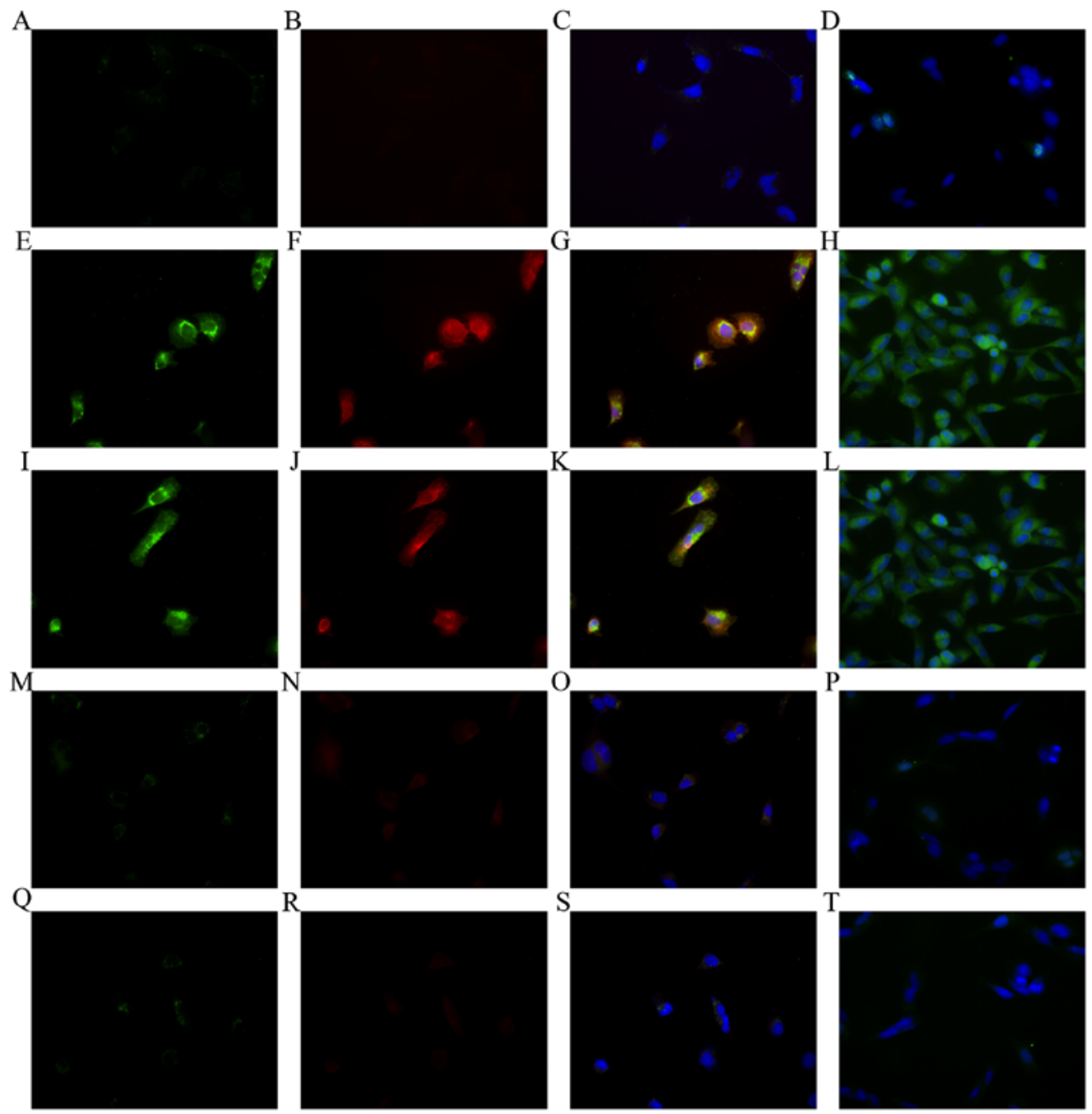

Figure 4. Immunofluorescent staining performed on MNNG/HOS cells before or after treatment. (A-D) Immunofluorescent staining performed on MNNG/ HOS cells without treatment. (A) Magnification (x400), stained for CD31. (B) Magnification (x400), for CD34. (C) Magnification (x400), the merging of A and B. (D) Magnification (x400), for vWF. (E-H) Immunofluorescent staining performed on MNNG/HOS cells exposed to hypoxia conditions with endothelial differentiation medium. (E) Magnification (x400), stained for CD31. (F) Magnification (x400), for CD34. (G) Magnification (x400), the merging of E and F. (H) Magnification (x400) for vWF. (I-L) Immunofluorescent staining performed on MNNG/HOS cells exposed to hypoxia conditions with essential medium. (I) Magnification (x400), stained for CD31. (J) Magnification (x400), for CD34. (K) Magnification (x400), the merging of G and H. (L) Magnification (x400), for vWF. (M-P) Immunofluorescent staining performed on MNNG/HOS cells exposed to normoxia conditions with endothelial differentiation medium. (M) Magnification (x400), stained for CD31. (N) Magnification (x400), for CD34. (O) Magnification (x400), the merging of E and F. (P) Magnification (x400) for vWF. (Q-T) Immunofluorescent staining performed on MNNG/HOS cells exposed to normoxia conditions with essential medium. (Q) Magnification (x400), stained for CD31. (R) Magnification (x400), for CD34. (S) Magnification (x400), the merging of E and F. (T) Magnification (x400) for vWF.

in cells cultivated on Matrigel under normoxia conditions, the normoxia and control group did not form tube structures even $96 \mathrm{~h}$ after seeding (Fig. 2).

Both the MRNA and protein levels of vascular endothelial cell (VECs) markers (vWF, CD31 and CD34) are increased after transdifferentiation. Since MNNG/HOS cells that were cultured under hypoxia conditions were able to form tubular-like structures and networks within Matrigel, we speculated that they may have the ability to transdifferentiate into VEC-like cells and have characteristics of VECs. Therefore, we examined the mRNA and protein levels of vWF, CD31 and CD34, which serve as cellular markers of VECs. The results showed that the level of mRNA of all the 3 cellular markers had significantly increased after MNNG/HOS cells were cultured under hypoxia conditions in the hypoxia and preconditioning group (Fig. 3). However, the levels of mRNA of all the 3 cellular markers were not significantly different between the nornoxia and control group (Fig. 3). In addition, to investigate the proteins of the 3 cell surface markers of VECs in osteosarcoma cells, we used immunofluorescent staining. Without treatment, few MNNG/HOS cells showed positive immunofluorescent staining for the 3 molecules (Fig. 4A-D). However, after exposure to hypoxic conditions, most MNNG/HOS cells in the hypoxia and preconditioning groups showed positive immunofluorescent staining for the 3 molecules (Fig. 4E-L). Few MNNG/HOS cells were positive for the 3 molecules under the condition of normoxia, for the normoxia and control group (Fig. 4M-T). The molecular change suggested that MNNG/HOS cells exposed to hypoxia may have a VEC phenotype. The results were consistent with the findings of RT-PCR.

Tumorigenesis and transdifferentiation into VEC-like cells in vivo. In order to examine the ability of MNNG/HOS cells to form endothelial vessels in vivo, MNNG/HOS cells in each 
A

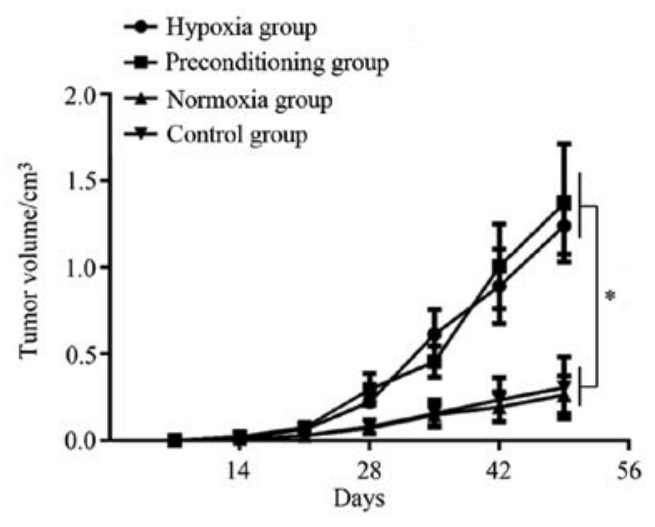

$\mathrm{C}$

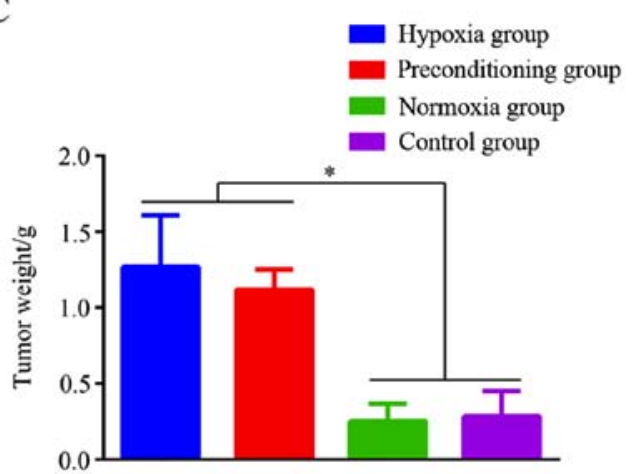

$\mathrm{B}$

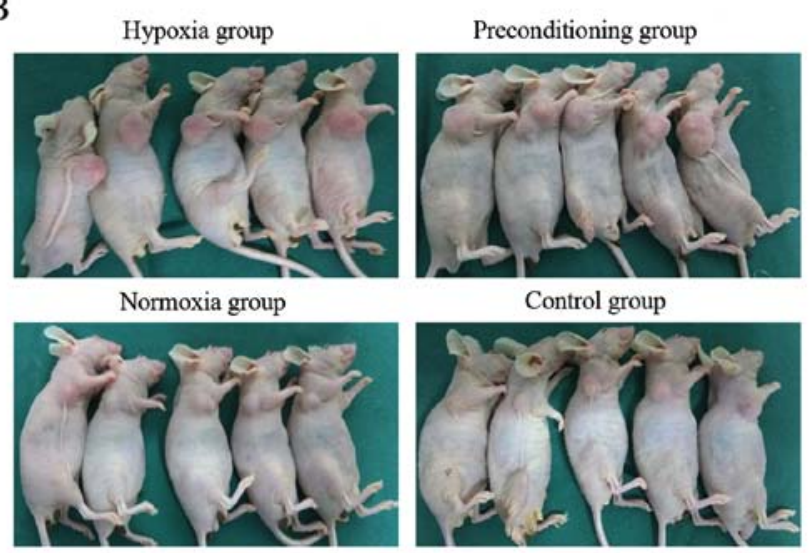

$\mathrm{D}$

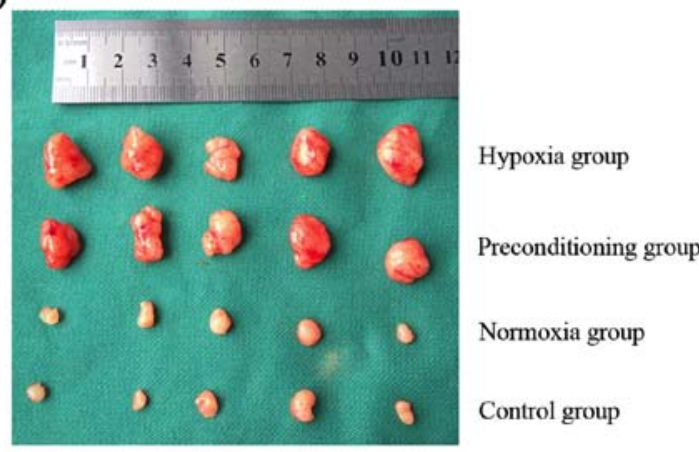

Figure 5. Hypoxia increases the proliferation and angiogenesis of MNNG/HOS cells in vivo. (A) The tumor growth curve of MNNG/HOS cells injected subcutaneously into nude mice demonstrated the effect of increased proliferation and angiogenesis of MNNG/HOS cells; " $\mathrm{P}<0.05$. (B) The MNNG/HOS xenografted tumors formed in hypoxia and preconditioning groups were larger than those in normoxia and control groups. (C) Tumor weight analysis after sacrifice demonstrated that the tumor weight was heavier in hypoxia and preconditioning groups than in normoxia and control groups; "P<0.05. (D) The quantity of vessels on the surface of MNNG/HOS xenografted tumors in hypoxia and preconditioning groups was greater than in normoxia and control groups.

A

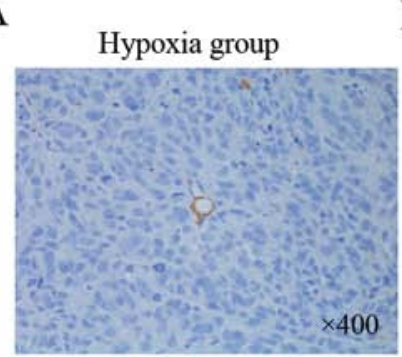

B

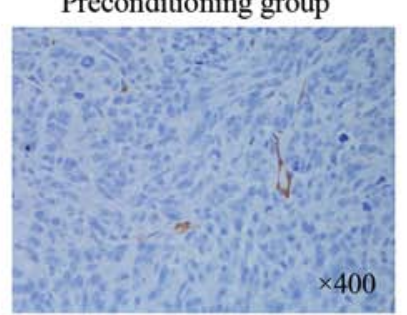

$\mathrm{C}$

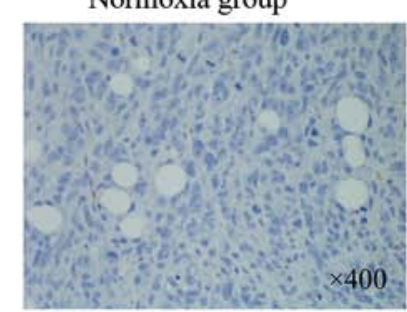

$\mathrm{D}$

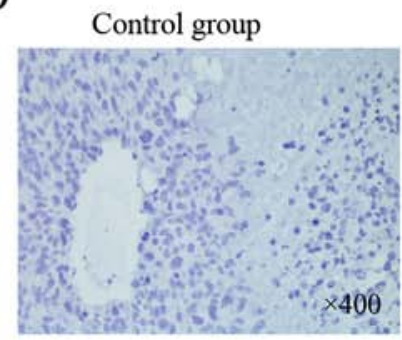

Figure 6. Immunohistochemical staining of MNNG/HOS xenografted tumors with anti-human CD31 to show the source of tumor blood vessels. Representative images show that the vessels in the MNNG/HOS xenografted tumors formed in the hypoxia and preconditioning groups originated from human osteosarcoma MNNG/HOS cells. The vessels in the MNNG/HOS xenografted tumors formed in the normoxia and control groups did not originate from human osteosarcoma MNNG/HOS cells.

group were subcutaneously injected into nude mice (day 0) and observed. The MNNG/HOS xenograft tumor volumes were calculated every 7 days, and weighed on day 49. As shown in Fig. 5A, there was no significant difference in the average volume of MNNG/HOS xenograft tumors among the 4 groups for the first 14 days. However, the volume of MNNG/HOS xenograft tumors in the hypoxia and preconditioning group were significantly increased after the first 14 days, compared with the control group $(\mathrm{P}<0.05)$. Nevertheless, there was no significant difference in the average volume of MNNG/HOS xenograft tumors between the normoxia and control group.
The MNNG/HOS xenograft tumors were weighed on day 49 , and the hypoxia and preconditioning group clearly produced larger tumors (Fig. 5B) and heavier tumors (Fig. 5C) compared with the control group. However, there was no difference in the average volume and weight of MNNG/HOS xenograft tumors between the normoxia and control group. Morphologically, the MNNG/HOS xenograft tumors in the hypoxia group and preconditioning hypoxia group clearly had a greater number of vessel structures compared with those in the control group (Fig. 5D). To study the origins of these blood vessels within the MNNG/HOS xenograft tumors in the 4 groups, 
we stained the tumor tissue with the anti-human CD31 antibody to distinguish whether the blood vessels originated from xenografted MNNG/HOS cells or from mouse VECs. The immunofluorescence results showed that the anti-human CD31-stained VECs were found only in MNNG/HOS xenograft tumors in the hypoxia and preconditioning group, but could not be found in those in the normoxia and control group (Fig. 6). These results from the in vivo studies further support the hypothesis that hypoxia plays an important role in inducing MNNG/HOS cell transdifferentiation into VEC-like cells.

\section{Discussion}

Hypoxia strongly stimulates an increase in the formation of new blood vasculature (16). In the present study, we examined whether MNNG/HOS cells can transdifferentiate into VEC-like cells both morphologically and functionally under the condition of hypoxia. We observed transdifferentiation of MNNG/HOS cells into VEC-like cells by cultivating the MNNG/HOS cells under hypoxia conditions with or without VEGF (an important angiogenic factor), and expression of several molecular markers of VECs (CD31, CD34 and vWF). When MNNG/HOS cells were cultured on Matrigel under hypoxia conditions, they gradually formed tubular-like structures. In addition, when MNNG/HOS cells were inoculated subcutaneously into BALB/c nude mice, blood vasculature derived from the MNNG/HOS cells was observed in xenograft tumors of the hypoxia group and the preconditioning group.

With sufficient blood supply, tumor cells can survive, proliferate and metastasize (17). Angiogenesis is an extremely important process facilitating the growth and metastasis of osteosarcoma (18). It also has become a major focus in tumor treatment (19). Angiogenesis is closely associated with the formation of vascular endothelial cells (VECs) (20). It is widely accepted that bone marrow-derived circulating endothelial precursors (CEPs) play a critical role in tumor-associated angiogenesis, the main source of the VECs (21). However, a recent study showed that bone marrow-derived CEPs do not contribute to the vascular endothelium (22). Moreover, recent studies suggest the possibility of tumor-derived endothelial cells in several malignant neoplasms, such as myeloma, breast cancer and neuroblastoma (10-14). In addition, research has shown that hypoxia can induce glioblastoma cells to transdifferentiate into VECs (15). Therefore, the transdifferentiation of MNNG/HOS cells into VEC-like cells under the condition of hypoxia is plausible.

Hypoxia plays a very important role in causing tumor angiogenesis (23), which is significantly correlated to tumor progression and poor outcome (24). Transcriptional responses to hypoxia are commonly regulated by hypoxia-inducible factors (HIFs). HIFs are composed of two subunits, HIF- $\alpha$ (an oxygen regulated subunit) and HIF- $\beta$. Yet, the traditionally accepted mechanism has been that hypoxia can activate VEGF, resulting in stimulation of endothelial cell proliferation, migration and assembly, and promotion of vessel sprouting and branching $(25,26)$. In the present study we found that MNNG/HOS cells can transdifferentiate into VEC-like cells under hypoxia conditions with or without VEGF. We also provide evidence that hypoxia can induce MNNG/HOS cells to transdifferentiate into VEC-like cells (Fig. 3), which may be a novel mechanism of neovascularization in human osteosarcoma tumors.

However, the mechanism of MNNG/HOS cell transdifferentiation into VEC-like cells under hypoxia conditions remains unclear. Research has shown that under hypoxic conditions, the malignant tumor cells can dedifferentiate into stem cell-like cells, such as neuroblastoma and breast cancer $(27,28)$. Moreover, recent research indicates the possibility of cancer stem cell differentiation into endothelial cells in some malignant tumors, such as glioma and ovarian tumors (29,30). Therefore, we think that the possible mechanism of MNNG/HOS cell transdifferentiation into VEC-like cells under hypoxia conditions may be that MNNG/HOS cells differentiate into stem cell-like cells under the condition of hypoxia, and then cancer stem cell-like cells differentiate into endothelial cells. This hypothesis deserves further study.

\section{Acknowledgements}

The present study was supported by the National Natural Science Foundation of China (no. 31571292).

\section{References}

1. Xu G, Kuang G, Jiang W, Jiang R and Jiang D: Polydatin promotes apoptosis through upregulation the ratio of $\mathrm{Bax} / \mathrm{Bcl}-2$ and inhibits proliferation by attenuating the $\beta$-catenin signaling in human osteosarcoma cells. Am J Transl Res 8: 922-931, 2016.

2. Cole HA, Ohba T, Ichikawa J, Nyman JS, Cates JM, Haro H, Schwartz HS and Schoenecker JG: Micro-computed tomography derived anisotropy detects tumor provoked deviations in bone in an orthotopic osteosarcoma murine model. PLoS One 9: e97381, 2014.

3. Longhi A, Errani C, De Paolis M, Mercuri M and Bacci G: Primary bone osteosarcoma in the pediatric age: State of the art. Cancer Treat Rev 32: 423-436, 2006.

4. Ren Z, Liang S, Yang J, Han X, Shan L, Wang B, Mu T, Zhang Y, Yang X, Xiong S et al: Coexpression of CXCR4 and MMP9 predicts lung metastasis and poor prognosis in resected osteosarcoma. Tumour Biol 37: 5089-5096, 2016.

5. Liu B, Yang H, Servaes S and Zhuang H: Solitary retroperitoneal metastasis as the initial site of the relapse of osteosarcoma revealed by FDG PET/CT. Clin Nucl Med 40: 892-894, 2015.

6. Hung TM, Cuong TD, Kim JA, Tae N, Lee JH and Min BS: Cassaine diterpene alkaloids from Erythrophleum fordii and their anti-angiogenic effect. Bioorg Med Chem Lett 24: 168-172, 2014.

7. Zhao C, Su Y, Zhang J, Feng Q, Qu L, Wang L, Liu C, Jiang B, Meng $L$ and Shou C: Fibrinogen-derived fibrinostatin inhibits tumor growth through anti-angiogenesis. Cancer Sci 106: 1596-1606, 2015.

8. Shojaei F, Wu X, Zhong C, Yu L, Liang XH, Yao J, Blanchard D, Bais C, Peale FV, van Bruggen N, et al: Bv8 regulates myeloidcell-dependent tumour angiogenesis. Nature 450: 825-831, 2007.

9. Tammela T, Zarkada G, Wallgard E, Murtomäki A, Suchting S, Wirzenius M, Waltari M, Hellström M, Schomber T, Peltonen R, et al: Blocking VEGFR-3 suppresses angiogenic sprouting and vascular network formation. Nature 454: 656-660, 2008.

10. Streubel B, Chott A, Huber D, Exner M, Jäger U, Wagner O and Schwarzinger I: Lymphoma-specific genetic aberrations in microvascular endothelial cells in B-cell lymphomas. N Engl J Med 351: 250-259, 2004.

11. Gunsilius E, Duba HC, Petzer AL, Kähler CM, Grünewald K, Stockhammer G, Gabl C, Dirnhofer S, Clausen J and Gastl G: Evidence from a leukaemia model for maintenance of vascular endothelium by bone-marrow-derived endothelial cells. Lancet 355: 1688-1691, 2000 .

12. Rigolin GM, Fraulini C, Ciccone M, Mauro E, Bugli AM, De Angeli C, Negrini M, Cuneo A and Castoldi G: Neoplastic circulating endothelial cells in multiple myeloma with 13 q14 deletion. Blood 107: 2531-2535, 2006. 
13. Soda Y, Marumoto T, Friedmann-Morvinski D, Soda M, Liu F, Michiue H, Pastorino S, Yang M, Hoffman RM, Kesari S, et al: Transdifferentiation of glioblastoma cells into vascular endothelial cells. Proc Natl Acad Sci USA 108: 4274-4280, 2011.

14. Bussolati B, Grange C, Sapino A and Camussi G: Endothelial cell differentiation of human breast tumour stem/progenitor cells. J Cell Mol Med 13: 309-319, 2009.

15. Pezzolo A, Parodi F, Corrias MV, Cinti R, Gambini C and Pistoia V: Tumor origin of endothelial cells in human neuroblastoma. J Clin Oncol 25: 376-383, 2007.

16. Griffith CK and George SC: The effect of hypoxia on in vitro prevascularization of a thick soft tissue. Tissue Eng Part A 15: 2423-2434, 2009.

17. Ren K, Yao N, Wang G, Tian L, Ma J, Shi X, Zhang L, Zhang J, Zhou X, Zhou G, et al: Vasculogenic mimicry: A new prognostic sign of human osteosarcoma. Hum Pathol 45: 2120-2129, 2014.

18. Peng N, Gao S, Guo X, Wang G, Cheng C, Li M and Liu K: Silencing of VEGF inhibits human osteosarcoma angiogenesis and promotes cell apoptosis via VEGF/PI3K/AKT signaling pathway. Am J Transl Res 8: 1005-1015, 2016.

19. Chen F, Chen L, He H, Huang W, Zhang R, Li P, Meng Y and Jiang X: Up-regulation of microRNA-16 in glioblastoma inhibits the function of endothelial cells and tumor angiogenesis by targeting Bmi-1. Anticancer Agents Med Chem 16: 609-620, 2016.

20. DeCicco-Skinner KL, Henry GH, Cataisson C, Tabib T, Gwilliam JC, Watson NJ, Bullwinkle EM, Falkenburg L, O'Neill RC, Morin A, et al: Endothelial cell tube formation assay for the in vitro study of angiogenesis.J Vis Exp 91: e51312, 2014.

21. Bertolini F, Shaked Y, Mancuso P and Kerbel RS: The multifaceted circulating endothelial cell in cancer: Towards marker and target identification. Nat Rev Cancer 6: 835-845, 2006.
22. Purhonen S, Palm J, Rossi D, Kaskenpää N, Rajantie I, Ylä-Herttuala S, Alitalo K, Weissman IL and Salven P: Bone marrow-derived circulating endothelial precursors do not contribute to vascular endothelium and are not needed for tumor growth. Proc Natl Acad Sci USA 105: 6620-6625, 2008.

23. Zhao X, Sun B, Liu Y, Zhang D, Liu Z, Zhao X, Gu Q, Han C, Dong $\mathrm{X}$, Che N, et al: Linearly patterned programmed cell necrosis induced by chronic hypoxia plays a role in melanoma angiogenesis. J Cancer 7: 22-31, 2016.

24. Wang W, Li GY, Zhu JY, Huang DB, Zhou HC, Zhong W and Ji CS: Overexpression of AGGF1 is correlated with angiogenesis and poor prognosis of hepatocellular carcinoma. Med Oncol 32: $131,2015$.

25. Uluer ET, Inan S, Ozbilgin K, Karaca F, Dicle N and Sanci $\mathrm{M}$ : The role of hypoxia related angiogenesis in uterine smooth muscle tumors. Biotech Histochem 90: 102-110, 2015.

26. Carmeliet P and Jain RK: Angiogenesis in cancer and other diseases. Nature 407: 249-257, 2000.

27. Bhaskara VK, Mohanam I, Rao JS and Mohanam S: Intermittent hypoxia regulates stem-like characteristics and differentiation of neuroblastoma cells. PLoS One 7: e30905, 2012.

28. Axelson H, Fredlund E, Ovenberger M, Landberg G and Påhlman S: Hypoxia-induced dedifferentiation of tumor cells - a mechanism behind heterogeneity and aggressiveness of solid tumors. Semin Cell Dev Biol 16: 554-563, 2005.

29. Zhao Y, Dong J, Huang Q, Lou M, Wang A and Lan Q: Endothelial cell transdifferentiation of human glioma stem progenitor cells in vitro. Brain Res Bull 82: 308-312, 2010.

30. Tang S, Xiang T, Huang S, Zhou J, Wang Z, Xie R, Long H and Zhu B: Ovarian cancer stem-like cells differentiate into endothelial cells and participate in tumor angiogenesis through autocrine CCL5 signaling. Cancer Lett 376: 137-147, 2016. 